\title{
MOTIVAÇÃO PARA TER SEXO EM ESTUDANTES DO ENSINO SUPERIOR
}

\author{
Paula Nelas \\ Adjunt Professor in Superior Health School \\ Polytechnic Institute of Viseu. PhD \\ pnelas@gmail.com \\ Emília Coutinho \\ Adjunt Professor in Superior Health School \\ Polytechnic Institute of Viseu. PhD \\ Cláudia Chaves \\ Adjunt Professor in Superior Health School \\ Polytechnic Institute of Viseu. PhD \\ Odete Amaral \\ Adjunt Professor in Superior Health School \\ Polytechnic Institute of Viseu. PhD \\ Carla Cruz \\ Adjunt Professor in Superior Health School \\ Polytechnic Institute of Viseu. PhD
}

Fecha de Recepción: 23 Febrero 2018

Fecha de Admisión: 10 Abril 2018

\section{RESUMO}

Enquadramento: 0 início da atividade sexual é um momento que tem impacto na vida dos jovens. É importante conhecer as motivações para a decisão de fazer sexo.

Objetivos: Determinar se a satisfação sexual (relação e confiança) influencia a motivação para fazer sexo.

Métodos: Trata-se de um estudo quantitativo, transversal, descritivo e correlacional, com uma amostra de 323 estudantes do ensino superior, com média de idade de 20,68 anos ( $d p= \pm 2,54$ anos). 0 instrumento de coleta de dados foi um questionário que possibilitou a caracterização sexual. A escala de satisfação com o relacionamento sexual (Ribeiro \& Raimundo, 2005) e escala de Motivação para ter ou não fazer sexo (Leal \& Maroco, 2010) também foram incluídas. Os procedimentos éticos foram garantidos. Os dados foram processados com o programa SPSS (Statistical Package for the Social Sciences) versão 24 para Windows.

Resultados: Na amostra $62.5 \%$ dos participantes tem namorado. Destes $53.2 \%$ namora há mais de dois anos. 0 namorado (30.7\%) e a mãe $(21.4 \%)$ são os interlocutores preferênciais sobre sexualidade. Na amostra $83.6 \%$ já iniciou a vida sexual. 0 sexo feminino $(63.2 \%)$ valoriza as inti- 


\section{MOTIVAÇÃO PARA TER SEXO EM ESTUDANTES DO ENSINO SUPERIOR}

midades próximas do coito. $54.2 \%$ do sexo feminino prefere não ter relações sexuais coitais com mais de uma pessoa. No atual relacionamento $69.2 \%$ tem relações sexuais e $81.7 \%$ faz contraceção. Os participantes revelam satisfação sexual positiva, tanto no relacionamento como na confiança. 0 namoro e a experiência sexual influenciam a satisfação sexual. Os homens apresentam o hedonismo, saúde e interdependência relacional como motivos para ter sexo. Os participantes sem experiência sexual referem o medo e o conservadorismo para não ter sexo. A satisfação sexual é preditora para ter sexo nas dimensões hedonismo e saúde, e interdependência relacional.

Conclusão: Propomos a criação de gabinetes nas universidades para esclarecimento de dúvidas e treino de competências relacionadas com a sexualidade, que proporcionem decisões sexuais que promovam a saúde sexual e reprodutiva.

Palavra-Chave: satisfação sexual; estudantes; ensino superior; sexualidade; motivação

\section{ABSTRACT}

\section{Motivation to have sex in higher education students.}

Background: The onset of sexual activity is a time that has an impact on young people's lives. It is important to know the motivations for the decision to have sex.

Objectives: To determine if sexual satisfaction (relationship and trust) influence the motivation to have sex.

Methodology: This is a quantitative, cross-sectional, descriptive and correlational study with a sample of 323 higher education students, with a mean age of 20.68 years ( $s d= \pm 2.54$ years). The data collection instrument was a questionnaire that enabled the construction of the sexual characterization. The satisfaction with the sexual relationship scale (Ribeiro \& Raimundo, 2005) and Motivation to have or not to have sex (Leal \& Maroco, 2010) was also included. The ethical procedures were guaranteed. The data was processed with the SPSS program (Statistical Package for the Social Sciences) version 24 for Windows.

Results: In the sample $62.5 \%$ of the participants have date. Of these $53.2 \%$ have been dating for more than two years. The boyfriend/girlfriend $(30.7 \%)$ and the mother $(21.4 \%)$ are the preferred interlocutors on sexuality. In the sample, $83.6 \%$ already started the sex life. The female $(63.2 \%)$ values intimacies close to intercourse. $54.2 \%$ of females prefer not to have sexual intercourse with more than one person. In the current relationship $69.2 \%$ have sex and $81.7 \%$ have contraception. Participants reveal positive sexual satisfaction in both relationship and trust. Dating and sexual experience influence sexual satisfaction. Male present hedonism, health, and relational interdependence as reasons for having sex. Participants without sexual experience refer fear and conservatism not to have sex. Sexual satisfaction is predictive of having sex in the dimensions of hedonism and health, and relational interdependence.

Conclusion: We propose the creation of offices in the universities to clarify doubts and to train sexuality-related skills that provide sexual decisions and promote sexual and reproductive health.

Keywords: sexual satisfaction; students; Higher education; sexuality; motivation

\section{INTRODUÇÃo}

A sexualidade assume um aspeto ímpar na vivência humana e as suas manifestações são socialmente adequadas e aceites, se enquadradas na cultura vigente (Antunes, 2007; Caetano, 2010). A Organização Mundial de Saúde (OMS) define a sexualidade como "uma energia que nos motiva para encontrar amor, contato, ternura e intimidade. Ela integra-se no modo como sentimos, movemos, tocamos e somos tocados, é ser-se sensual e ao mesmo tempo ser-se sexual. A sexualidade influencia pensamentos, sentimentos, ações e interações e, por isso, influência também a nossa saúde física e mental" (Nelas, P., Fernandes, C., Ferreira, M., Duarte, J. \& Chaves, C. 2010). Assim, a sexua- 
lidade configura-se, enquanto fenómeno inerente ao desenvolvimento humano, compreendida como conceito amplo que abrange as relações afetivas e sexuais durante todo o desenvolvimento humano. (Orti, N.P., Souza, V.B., Maia, A.C.B., 2010).

A visão contemporânea reconhece a sexualidade humana como uma realidade complexa que envolve a procriação, o amor e o prazer, tendo três áreas fundamentais de expressão, a anátomofisiológica, a afetivorelacional e a social, não sendo desvalorizado o envolvimento emocional, a ternura e o erotismo (Antunes, 2007).

No ambiente universitário, os relacionamentos ocorrem entre jovens provenientes de diversas cidades, cada uma transportando a cultura familiar, onde se incluem os padrões morais, com os respetivos tabus e crenças sexuais. Deste modo, surgem os novos modelos do que seja sexualidade, dos comportamentos e das atitudes tomadas em relação ao sexo, das preferências manifestadas entre os pares, enfim, um processo de adaptação que pode durar dias ou meses, dependendo das questões familiares, morais, religiosas que determinaram o modo de significar elaborado por cada jovem. (Rangel \& Queiroz, 2008). Assim, as relações íntimas que se desenvolvem neste período, de amizade ou namoro, estabelecem um laço que confere segurança emocional neste momento de distanciamento das relações parentais.

Apesar de a maioria dos jovens já ter iniciado a vida sexual quando ingressa no ensino superior, esta continua a ser uma área de exploração e investimento. A valorização da procura de sensações novas, do prazer, da experimentação e até de algumas transgressões e atitudes não convencionais pode sobressair pela força do estilo de vida emergente (Dalton \& Galambos, 2009). Assim, comportamentos, outrora reprimidos e socialmente reprovados, como o sexo pré-conjugal, passaram a ser aceites e considerados naturais e até positivos na vivência de uma sexualidade plena e saudável (Antunes, 2007).

Diversos autores têm aludido a um processo de mudança sociocultural no sentido de uma maior valorização da sexualidade e do erotismo, de uma maior permissividade em relação às expressões e aos comportamentos sexuais, nomeadamente na maior aceitação da diversidade inerente à sexualidade juvenil (Vilar, Ferreira, \& Duarte, 2009). A par da fidelidade, ideologia tradicional do amor romântico, as relações sexuais juvenis passaram a caracterizar-se por uma certa favorabilidade do sexo ocasional, embora atualmente se assista à junção de ambas no seio das relações sexuais socialmente aceites (Antunes, 2007).

As atitudes sexuais são influenciadas por aspetos culturais, interpessoais e psicológicos provenientes de esquemas complexos, dependendo das normas da sociedade, das estratégias de relacionamento e da forma como a pessoa lida com a sexualidade (Davidson, Moore, Earle \& Davis, 2008). Estas, apreendem-se ao longo da vida resultado da influência externa e crenças ou valores consideradas relevantes. Por outro lado, a própria pessoa realiza um esforço integrativo, ajustandose aos que a rodeiam.

0 comportamento sexual resulta da interação de aspetos biológicos relacionados com a procura da satisfação como elemento motivador para a ação, aspetos cognitivos nomeadamente as ideias e crenças subjacentes às atitudes e aspetos afetivos que vão modular toda a atividade sexual entre duas pessoas. Por fim, as consequências do comportamento vão influenciar cada uma destas componentes regulando comportamentos futuros. No comportamento sexual estão implícitos estímulos externos ou eróticos, que podem ser pré-programados e adquiridos, e como tal, não poderá ser entendido apenas num ato mecânico que visa a satisfação de um instinto. E no que respeita aos processos afetivos é o desejo, a atração e 0 enamoramento que intervêm na expressão dos comportamentos sexuais (Lopez \& Fuertes,1999).

A intimidade encontra-se estreitamente ligada às relações, e é definida como um estado subje- 


\section{MOTIVAÇÃO PARA TER SEXO EM ESTUDANTES DO ENSINO SUPERIOR}

tivo de proximidade a uma outra pessoa gratificante, um desejo de calor e de relacionamento que fornece a oportunidade de expressão, motivados pela sexualidade (Pinto, 2009). As capacidades de interação cognitiva, comportamental e afetiva são a base da intimidade e afetam a capacidade e a motivação para se ser íntimo. (Pinto, 2009). 0 amor e a intimidade influenciam-se mutuamente, de tal modo que a intimidade é um dos processos afetivos que estimula e é incentivada pelo amor (Narciso, Costa \& Prata, 2002). Já no que diz respeito aos processos afetivos é o desejo, a atração e o namoro que intervêm na expressão dos comportamentos sexuais.

As necessidades, motivos, objetos ligados à intimidade influenciam o modo como as pessoas abordam a intimidade. Pode haver variações na força de necessidade ou desejo de intimidade. Estas diferenças, são indicadores de uma variedade de comportamentos interpessoais (Pinto, 2009). Apesar de ser possivel viver experiências de intimidade em vários tipos de relações interpessoais, é nas relações amorosas que esta é mais desejada. Estas, caracterizam-se por terem um grau elevado de intensidade e importância em comparação com outras relações íntimas (Collins, Welsh, \& Furman, 2009).

Atualmente, são vários os estudos que apontam a satisfação sexual como um fator de impacto relevante na satisfação e na perceção do relacionamento (Dunn, Croft \& Hackett, 2000). As atitudes e expectativas sexuais dos parceiros, os seus desejos e as atividades que empreendem em conjunto têm consequências significativas a nível individual e a nível do relacionamento (Sprecher \& Regan, 2000).

São vários os estudos que apontam a satisfação sexual como um fator de impacto relevante na satisfação e na perceção do relacionamento (Dunn, Croft \& Hackett, 2000). Esta, é um dos fatores psicológicos avaliados no âmbito da sexualidade, que corresponde ao grau no qual a atividade sexual de uma pessoa corresponde aos seus ideais. Mencionam ainda que a perceção de satisfação com a vida sexual encontra-se intrinsecamente relacionada com as experiências sexuais passadas do indivíduo, expetativas atuais e aspirações futuras (Pechorro, Diniz, \& Vieira, 2009). Em geral, a satisfação sexual tem sido positivamente relacionada com a satisfação conjugal ou qualidade do relacionamento (Pechorro, P., Diniz, A., \& Vieira, R., 2009).

A motivação sexual humana vai para além do instinto sexual. É constituída por uma panóplia complexa de fatores (hormonais, cognitivos, educacionais e culturais) que se correlacionam entre si (Oliveira, 2011). Os impulsos que levam uma pessoa a ter atividade sexual são, da mesma forma que 0 desejo, multifacetados, ou seja, envolvem o sexo, a carência afetiva, necessidade de atenção, de carinho, exercício de poder, dentre outros, cada vez mais afastado da meta reprodutiva (Andrade, 2009). 0 prazer resultante do ato sexual diferencia o Homem dos restantes animais. Ele é o único ser que, objetivamente, tem relações sexual pelo prazer, entre outros motivos, e não apenas com a finalidade reprodutiva (Oliveira, 2011).

No final dos anos oitenta foram documentados sete motivos para ter sexo: por puro prazer, para expressar uma proximidade emocional, para reproduzir, porque um dos parceiros o deseja, para agradar o parceiro, para fazer uma conquista e para aliviar a tensão sexual. Posteriormente, Leal e Maroco (2010) consideraram cinco dimensões motivacionais da sexualidade: interdependência relacional, hedonismo, normatividade, paixão e reprodução. Assim a expressão da sexualidade, continua a ser uma realidade social que envolve polémicas e contradições. Se por um lado o impulso sexual é fonte de motivação, por outro, o sistema de crenças interiorizado na sociedade limita a legitimidade dessas condutas.

Por conseguinte, consideramos pertinente e relevante 0 desenvolvimento de estudos sobre as motivações que estão na base dos comportamentos sexuais dos jovens universitários e, em particular, o que os impele a fazer ou não fazer sexo. 


\section{METODOLOGIA}

0 estudo realizado é quantitativo, longitudinal, descritivo e correlacional, com uma amostra não probabilística por conveniência, sendo critério ser estudante do ensino superior. A amostra perfaz 323 participantes, 244 do sexo feminino e 79 do sexo masculino. A média de idade é de 20.68 anos ( $d p \pm 2.54)$. Todos os procedimentos éticos e legais foram assegurados. Os dados foram tratados com recurso ao SPSS versão 24.0 para Windows.

0 instrumento de recolha de dados foi o questionário dividido em 2 partes. A primeira permite fazer a caraterização sociodemográfica (sexo, idade e local de residência), a segunda parte permite fazer a caraterização sexual (relação de namoro, tempo de namoro, interlocutor preferencial sobre sexualidade, experiência sexual, uso de contraceção, idade da primeira relação sexual, relações sexuais atuais e relacionamento íntimo. A terceira parte inclui a Escala de satisfação com o relacionamento sexual (avalia as dimensões relacionamento sexual e confiança) (Ribeiro \& Raimundo, 2005) e a Escala de motivação para fazer ou não fazer sexo (avalia as dimensões hedonismo/saúde, interdependência relacional, medo e conservadorismo) (Leal \& Maroco, 2010).

\section{RESULTADOS}

A amostra é constituída por $24.5 \%$ de participantes do sexo masculino e $75.5 \%$ do sexo feminino. Da totalidade dos participantes $40.2 \%$ reside em meio rural e $50.8 \%$ em meio urbano.

Dos estudantes que namoram $16.7 \%$ são do sexo masculino e $45.8 \%$ são do sexo feminino. 0s que namoram, $53.2 \%$ fazem-no há mais de dois anos, $30.5 \%$ de seis meses a dois anos e $16.3 \%$ há menos de meio ano. 0 namorado é o interlocutor preferencial sobre sexualidade (30.7\%), seguido da mãe (21.4\%) e os amigos (15.2\%). Na amostra, 83.6\% já teve experiência sexual. Existe uma tendência no sexo feminino para ter relações sexuais em média aos 17.48 anos e 0 sexo masculino aos 16.3 anos. Há uma tendência no sexo feminino (63.2\%) para valorizar as intimidades, próximas do coito, incluindo carícias diretas em qualquer parte do corpo incluindo genitais. Há uma tendência no sexo feminino (54.2\%) para não terem relações sexuais coitais com mais de uma pessoa. $69.2 \%$ tem relações sexuais no atual relacionamento amoroso. Destes, $47.7 \%$ iniciou as relações após seis meses de relacionamento. Na amostra, 81.7\% faz contraceção, preferencialmente a pílula contracetiva (65.2\%). Os participantes que recorreram à contraceção de emergência (18.9\%), dizem tê-lo feito pelo menos uma vez $77 \%$.

Os participantes revelam uma satisfação sexual positiva, tanto ao nível do relacionamento como na confiança. 0 sexo e a idade, não influenciam a satisfação sexual. A satisfação sexual é superior nos participantes que namoram, com experiência sexual e com relações sexuais no atual relacionamento.

Relativamente à motivação para ter vou não ter sexo, foram encontradas diferenças estatisticamente significativas entre o sexo masculino e feminino na subescala hedonismo e saúde e interdependência relacional, com valores mais elevados no sexo masculino. Na subescala medo e conservadorismo não são evidentes diferenças significativas entre sexos. Os participantes com idades superiores a 22 anos estão mais motivados para ter sexo com motivos relacionados com o hedonismo e saúde e menos motivos por razões ligadas ao conservadorismo. Os que ainda não tiveram relações sexuais apresentam o medo e o conservadorismo como motivo. Os participantes com intimidades próximas do coito referem como motivos para ter sexo o hedonismo e saúde e a interdependência relacional. Os que têm relações sexuais no atual relacionamento, referem como motivo para ter sexo a interdependência relacional.

É fator preditor para ter sexo, relacionado com a dimensão hedonismo e saúde, as dimensões relacionamento sexual e confiança, da escala satisfação com o relacionamento sexual. É ainda pre- 


\section{MOTIVAÇÃO PARA TER SEXO EM ESTUDANTES DO ENSINO SUPERIOR}

ditor para ter sexo, para a dimensão interdependência relacional, a confiança. Não foi encontrada associação entre não ter sexo e a satisfação com o relacionamento sexual, nas dimensões em análise (relacionamento e confiança).

\section{DISCUSSÃO DOS RESULTADOS}

A maioria dos participantes $(62,5 \%)$ tem um relacionamento. Damos destaque a estudo de Matos, Reis, Ramiro \& Equipa Aventura Social (2011) com estudantes do ensino superior sobre saúde sexual e reprodutiva onde a maior parte dos estudantes $(60,6 \%)$ refere ter um relacionamento amoroso.

Os participantes do sexo masculino e os do feminino têm diferentes interlocutores sobre assuntos de sexualidade. 0 estudo de Matos, Simões, Tomé, Camacho, Ferreira, Ramiro, Reis, Diniz, Gaspar, Veloso, Loureiro, Borges \& Equipa Aventura Social (2010), relativamente ao interlocutor sobre sexualidade, destaca os amigos $(65,9 \%)$, a internet $(48,7)$, a comunicação social $(46,7)$ e os profissionais de saúde $(40,7 \%)$. As mulheres mais frequentemente obtêm informação com profissionais de saúde $(47,5 \%)$ e os homens na Internet $(53,7 \%)$.

$\mathrm{Na}$ amostra, $83.6 \%$ já teve experiência sexual. Existe uma tendência no sexo feminino para ter relações sexuais em média aos 17.48 anos e 0 sexo masculino aos 16.3 anos. 0 estudo realizado em 2010 à população universitária portuguesa por Matos, Reis, Ramiro e Equipa Aventura Social, refere que a maioria é sexualmente ativa $(83,3 \%)$ e são os homens que mais frequentemente mencionam ter tido relações sexuais $(88,6 \%)$. Também, são os homens que iniciam a atividade sexual mais cedo (28\%) em comparação com as mulheres (17,4\%). A literatura científica sugere que a primeira relação sexual é considerada uma marca na vida dos jovens e no contexto português verificase, cada ver mais precocemente. Na comparação da idade da primeira relação sexual entre os estudantes verifica-se que há uma tendência no sexo feminino para ter relações sexuais mais tarde (17 anos) e no sexo masculino mais cedo (16 anos).

Na análise das intimidades, próximas do coito, incluindo carícias diretas em qualquer parte do corpo incluindo genitais verifica-se que há uma tendência no sexo feminino para valorizar este tipo de intimidades. 0 estudo de Matos et al. em 2010 refere que são os homens que dão mais importância às caricias genitais, coito e orgasmo para obtenção de prazer na relação $(96,8 \%)$ comparativamente às mulheres $(93,6)$. Nos dados relativos às relações sexuais coitais com mais de uma pessoa, verifica-se que no sexo masculino já tiveram relações sexuais coitais com mais de uma pessoa. Há uma tendência no sexo masculino (9.6\%) para terem relações sexuais ao fim de 1 mês e no sexo feminino depois de 6 meses (39\%). Deste modo e à semelhança do nosso estudo, 0 estudo realizado por Matos et al. (2010) refere que são os homens que mais frequentemente tiveram relações sexuais uma semana ou menos depois de iniciarem o relacionamento amoroso $(30,4 \%)$ e as mulheres mais frequentemente levaram três a seis meses (18,3\%) e seis meses a um ano (16,2\%).

No sexo masculino a maior percentagem (22\%) faz contraceção com preservativo e no sexo feminino é feita com a pílula (62.9\%). Os resultados mostram que alguns participantes dão pouca importância ao uso de contraceção de barreira, pois alguns participantes não gostam de usar preservativo pela possibilidade de diminuição do prazer $(6.4 \%)$ ou por não 0 terem à mão $(5.6 \%)$. Também as participantes do sexo feminino referem diminuição do prazer $(24.8 \%)$ e porque não 0 têm à mão (15.2\%). Evidencia-se uma tendência masculina de não usar preservativo porque o parceiro usa outro contracetivo. Estes resultados são similares aos de Ribeiro e Fernandes (2009) sobre os motivos para usar ou não usar preservativo nas relações sexuais dos estudantes do ensino superior, os jovens que já iniciaram a sua vida sexual, $54,5 \%$ não usam preservativo porque confiam no parceiro ou porque praticam sexo com o mesmo companheiro $(54,4 \%)$, referem não ter 
usado preservativo por não quererem ou não gostarem $24,3 \%$ e não usaram porque não tinham à mão no momento do ato sexual $(24,4 \%)$.

A maior parte das participantes que recorreu à contraceção de emergência refere que 0 fez pelo menos uma vez (77\%). Uma intervenção regular e sistematizada, adaptada à população jovem, por parte dos profissionais de saúde deve incentivar e potenciar práticas contracetivas cada vez mais adequadas.

Quando se fala de sexualidade é importante referenciar a satisfação sexual interpretada mediante a análise do grau de satisfação face ao relacionamento e à confiança. Assim, os participantes revelam um grau de satisfação sexual positivo, tanto no relacionamento como na confiança. Podem observar-se valores mais elevados no sexo masculino e com idades acima dos 22 anos, contudo, as diferenças não são estatisticamente significativas entre sexos. Também a satisfação sexual dos participantes que namoram, com experiência sexual e com relações sexuais no atual relacionamento é mais elevada. No estudo realizado por Matos, M. G., Simões C., Tomé G., Camacho I., Ferreira M., Ramiro L.,... Equipa Aventura Social (2010), refere que a maioria dos estudantes do ensino superior está muito satisfeito sexualmente $(96,2 \%)$. As mulheres e os participantes com um relacionamento há mais de um ano referem um grau de satisfação maior.

0 hedonismo e saúde e a interdependência relacional são preconizados como motivos para fazer sexo, enquanto o medo, o conservadorismo, como motivos para não fazer sexo, de acordo com Leal e Maroco (2010). No nosso estudo, os participantes do sexo masculino manifestam como motivos para ter sexo o hedonismo e saúde e a interdependência relacional, com diferenças estatísticas significativas quando comparados com o outro sexo. De facto, existem diferenças entre a motivação sexual dos homens e mulheres, sendo as mulheres mais motivadas pela busca da estabilidade e realização de vínculo estável, enquanto os homens estão mais motivados pelo hedonismo. 0 estudo Saúde Sexual e Reprodutiva dos Estudantes do Ensino Superior, realizado por Matos, M. G., Reis, M. Ramiro, L., \& Equipa Aventura Social (2011), concluíram que são os homens que mais tomam a iniciativa para ter relações sexuais. Relativamente aos motivos para não ter sexo (medo e conservadorismo), Leal e Maroco, (2010) apresentam como motivos o facto de, quererem manter a virgindade, por não terem encontrado a pessoa certa e pelo desejo de casar virgem.

Os participantes com idades superiores a 22 anos apresentam motivos para ter sexo relacionado com o hedonismo e saúde e como motivos para não 0 ter relacionado ao conservadorismo. No estudo de Oliveira (2011) os motivos relacionados com hedonismo e saúde parecem ser os que motivam os adolescentes mais velhos para fazer sexo. Outros estudos semelhantes incidem também sobre as faixas etárias da adolescência. Os participantes com experiência sexual estão mais motivados por questões relacionadas com o hedonismo e saúde, e os que têm relações sexuais no atual relacionamento têm mais motivos associados à interdependência relacional. Esta interdependência relacional é mais comum entre o sexo feminino, como também defende Matos, Reis, Ramiro \& Equipa Aventura Social (2012), quando indica que, no relacionamento amoroso, as mulheres dão mais importância aos sentimentos e comunicação na relação.

\section{CONCLUSÕES}

Homens e mulheres diferem no modo como vivenciam a sexualidade, sofrendo influência marcante do contexto sociocultural. Os profissionais de saúde devem ser sensiveis à diversidade da vivência da sexualidade. Devem sensibilizar a comunidade no geral e os jovens adultos em particular para a prevenção de comportamentos de risco. Face aos resultados propomos a criação de gabinetes de esclarecimento nas universidades, constituídas por equipas pluridisciplinares, com recursos às tecnologias de informação recentes, para esclarecimento e treino de competências relacio- 


\section{MOTIVAÇÃO PARA TER SEXO EM ESTUDANTES DO ENSINO SUPERIOR}

nadas com a sexualidade, pois para que o jovem adulto opte por comportamentos sexuais seguros, tem que ser detentor de informação suficiente e de qualidade que permita decidir com segurança. Assim, como estratégias pedagógicas sugerimos que devem ser implementadas metodologias ativas e participativas que promovam a aproximação dos profissionais de saúde aos jovens de forma a contribuir com uma orientação adequada, para que estes possam tomar conhecimento de como vivenciar uma sexualidade consciente, favorecendo atitudes responsáveis e efetivas na construção de uma identidade consciente e autónoma, prevenindo comportamentos de risco.

\section{AGRADECIMENTOS}

Este trabalho é financiado por fundos nacionais através da FCT - Fundação para a Ciência e a Tecnologia, I.P., no âmbito do projeto UID/Multi/04016/2016. Agradecemos adicionalmente ao Instituto Politécnico de Viseu e ao CI\&DETS pelo apoio prestado.

\section{BIBLIOGRAFIA}

Andrade, A. L., Garcia, A., \& Cano, D. S. (2009). Preditores da satisfação global em relacionamentos românticos. Psicologia: Teoria e prática, 11(3), 143-156. Brasil. Acedido em http://pepsic.bvsalud.org/scielo.php?pid=S1516-36872009000300012\&script=sci_arttext

Antunes, M. T. C. (2007). Atitudes e comportamentos sexuais de estudantes do ensino superior. Coimbra: Formasau, Lda.

Caetano, J. A. M. (2010). Sexualidade, saúde e direitos humanos. In F. Teixeira, I. P. Martins, P. R. M. Ribeiro, I. Chagas, A. C. B. Maia, T. Vilaça,... M. M. Melo (Orgs.), Sexualidade e educação sexual: políticas educativas, investigação e práticas. (Secção I, pp. 3-29). Braga: Edições CIEd: Centro de investigação em Educação, Universidade do Minho. Acedido em http://www.fpccsida.org.pt/images/stories/Livro_I_CISES.pdf

Collins, W. A., Welsh, D. P., \& Furman, W. (2009). Adolescent romantic relationships. Annual Review of Psychology, 60, 631-652.

Dalton, A., \& Galambos, N. (2009). Affect and sexual behavior in the transition to University. Archives of Sexual Behavior, 38(5), 675-687. Acedido em http://www.ncbi.nlm.nih.gov/pmc/articles/PMC3760485/

Davidson, J. K. S., Moore, N. B., Earle, J. R., \& Davis R. (2008). Sexual attitudes and behavior at four universities: Do region, race and/or religion matter?. Adolescence, 43(170), 189-220.

Dunn, K. M., Croft, P. R., \& Hackett, G. I. (2000). Satisfaction in the sex life of a general popuIation sample. Journal of Sex Marital Therapy, 26(2), 141-151. Acedido em http://www.ncbi.nlm.nih.gov/pubmed/10782446

Leal, I., \& Maroco, J. (2010). Avaliação em sexualidade e parentalidade. Rio de Janeiro: Editora Legis.

Lopez, F., \& Fuertes, A. (1999). Para compreender a sexualidade. Lisboa: Bertrand.

Matos, M. G., Reis M., Ramiro L., \& Equipa Aventura Social. (2012). A saúde sexual e reprodutiva dos estudantes universitários: Relatório de estudo, Dados de 2010. Lisboa: Centro de Malária e Outras Doenças Tropicais/IHMT/UNL. Acedido em http://aventurasocial.com/arquivo/1356031665_V5\%20Relatorio-Universitarios_protegido.pdf

Matos, M. G., Reis, M. Ramiro, L., \& Equipa Aventura Social. (2011). Saúde sexual e reprodutiva dos estudantes universitários: Relatório do estudo - Dados Nacionais 2010. Lisboa: Centro de Malária e Outras Doenças Tropicais/IHMT/UNL. Acedido em http://aventurasocial.com/arquivo/1303148036_Relatorio_HBSC_SSREU.pdf

Matos, M. G., Simões C., Tomé G., Camacho I., Ferreira M., Ramiro L.,... Equipa Aventura 
Social. (2010). Aventura social \& saúde: A saúde dos adolescentes portugueses: Relatório do estudo HBSC 2010. Lisboa. Acedido em http://aventurasocial.com/arquivo/ 1303144700_Relatorio_HBSC_adolescentes.pdf

Narciso, I., Costa, M. P., \& Prata, F. (2002). Intimidade e compromisso pessoal ou "aquilo que pode fazer com que um casamento funcione". Revista Portuguesa de Psicologia, 36, 67-88.

Nelas, P., Fernandes, C., Ferreira, M., Duarte, J. \& Chaves, C. (2010). Construção e validação da escala de atitudes face à sexualidade em adolescentes (AFSA). In F. Teixeira, I. P. Martins, P. R. M. Ribeiro, I. Chagas, A. C. B. Maia, T. Vilaça,... S. M. M. Melo (Orgs.), Sexualidade e educação sexual: Políticas educativas, investigação e práticas. (Secção IV, pp. 180-184). Braga: Edições CIEd: Centro de investigação em Educação, Universidade do Minho. Acedido em http://www.fpccsida.org.pt/images/stories/Livro_I_CISES.pdf

Oliveira, V. C. M. (2011). Sexualidade adolescente: Motivação para fazer ou não fazer sexo (Dissertação de mestrado, Instituto Politécnico de Viseu). Acedido em http://repositorio.ipv.pt/handle/10400.19/1572

Orti, N. P., Souza, V. B., \& Maia, A. C.B. (2010). Representação de género no relato de crianças abrigadas. In F. Teixeira, I. P. Martins, P. R. M. Ribeiro, I. Chagas, A. C. B. Maia, T. Vilaça,... S. M. M. Melo (Orgs.), Sexualidade e educação sexual: Políticas educativas, investigação e práticas. (Secção I, pp. 23-29). Braga: Edições CIEd: Centro de investigação em Educação, Universidade do Minho. Acedido em http://www.fpccsida.org.pt/images/stories/Livro_I_CISES.pdf

Pechorro, P., Diniz, A., \& Vieira, R. (2009). Satisfação sexual feminina: Relação com funcionamento sexual e comportamentos sexuais. Análise Psicológica, 27(1), 99-108. Acedido em http://www.scielo.oces.mctes.pt/pdf/aps/v27n1/v27n1a08.pdf

Pinto, M. C. (2009). Intimidade em adolescentes de diferentes grupos etários. Lisboa: Altocomissariado para a imigração e diálogo intercultural. Acedido em http://www.oi.acidi.gov.pt/docs/Colec_Teses/tese_25.pdf

Rangel, D. L. 0., \& Queiroz, A. B. A. (2008). A representação social das adolescentes sobre a gravidez nesta etapa de vida. Esc Anna Nery, Revista de Enfermagem, 12 (4), 780-788. Acedido em http://www.scielo.br/pdf/ean/v12n4/v12n4a24.pdf

Ribeiro, J., \& Raimundo, A. (2005). Estudo de adaptação do Questionário de Satisfação com 0 Relacionamento Sexual (QSRS) em mulheres com incontinência urinária. Psicologia, Saúde \& Doenças, 6(2), 191-202.

Ribeiro, M. I. B., \& Fernandes, A. J. G. (2009). Comportamentos sexuais de risco em estudantes do ensino superior público da cidade de Bragança. Psicologia, Saúde \& Doenças, 10 (1), 99-113. Acedido em http://www.scielo.mec.pt/pdf/psd/v10n1/v10n1a08.pdf

Sprecher, S., \& Regan, P. M. (2000). Sexuality in a relational context. In C. Hendrick, \& S. S. Hendrick (Eds.), Close relationships: A sourcerbook (cap. 16, pp. 218-226). California: SAGE Publications, Inc.

Vilar, D., Ferreira, P., \& Duarte, S.A. (2009). Educação sexual dos jovens portugueses: Conhecimentos e fontes. Revista Educação Sexual em rede, 5, 2-53. Acedido em http://www.apf.pt/?area=002\&mid=004\&sid=004 
